Relations industrielles

Industrial Relations

\title{
Supiot, Alain (sous la direction de), Au-delà de l'emploi - Transformations du travail et devenir du droit du travail en Europe
}

\section{Pierre Verge}

Volume 54, numéro 2, 1999

URI : https://id.erudit.org/iderudit/051242ar

DOI : https://doi.org/10.7202/051242ar

Aller au sommaire du numéro

Éditeur(s)

Département des relations industrielles de l'Université Laval

ISSN

0034-379X (imprimé)

1703-8138 (numérique)

Découvrir la revue

Citer ce compte rendu

Verge, P. (1999). Compte rendu de [Supiot, Alain (sous la direction de), Au-delà de l'emploi - Transformations du travail et devenir du droit du travail en Europe]. Relations industrielles / Industrial Relations, 54(2), 416-418.

https://doi.org/10.7202/051242ar

Tous droits réservés @ C Département des relations industrielles de l'Universite Laval, 1999
Ce document est protégé par la loi sur le droit d'auteur. L’utilisation des services d’Érudit (y compris la reproduction) est assujettie à sa politique d'utilisation que vous pouvez consulter en ligne.

https://apropos.erudit.org/fr/usagers/politique-dutilisation/ 
this variation seems to reflect the extent to which the authors have followed the editors' guidelines to cut back on historical material and concentrate on recent changes.

I have only a few minor "quibbles" about this book. A useful, but difficult, exercise would have been a concluding chapter seeking to summarize and distil what had gone before, although the editors' introduction does this to some extent. Secondly, a little stronger editorial control might have provided more of a common framework of discussion for individual chapters, as some rather "talk past each other". Thirdly, a little more emphasis on pan-European pressures and developments would have been welcomed in some chapters; EU-initiated develop- ments such as European works councils rarely got a mention in some cases.

However, there is no real question that this book provides the best, up-todate overview of changing European industrial relations at the present time. This edition does not cover the countries of Eastern Europe where things have not yet fully settled down; this being said, the German chapter does have some very useful material and insights on the East German scene. I, for one, hope and expect to see a third edition along these lines.

P. B. BEAUMONT

Department of Management Studies University of Glasgow

\section{Au-delà de l'emploi - Transformations du travail et devenir du droit du travail en Europe}

Rapport pour la Commission européenne sous la direction d'Alain SUPIOT, Paris : Flammarion, 1999, 321 p., ISBN 2-08212526-2.

Il s'agit de la version en langue française d'un rapport présenté à la Commission européenne par un groupe de neuf experts, de différentes nationalités européennes, dont le professeur Alain Supiot de l'Université de Nantes, rapporteur général. Ils avaient pour tâche de "réfléchir de manière prospective et constructive au niveau communautaire et dans un cadre interculturel et interdisciplinaire sur l'avenir du travail et du droit du travail [...] ». L'approche est à la fois transnationale - le rapport se fonde principalement sur les informations et les analyses des situations nationales correspondant à la nationalité des experts, transdisciplinaire - le droit ouvert sur une réalité sociale, qu'il contribue lui-même à transformer et diachronique - l'approche se veut soucieuse de replacer le changement dans sa perspective historique.

L'ampleur du sujet confère un intérêt particulier aux angles choisis pour l'appréhender. Les "percées " annoncées (p. 21) sont les suivantes: travail et pou- voir privé ; travail et statut professionnel ; travail et temps; travail et organisation collective et travail et pouvoirs publics. Lecture faite, il faut convenir de la réalité de leur interrelation. 11 en va de même de deux autres chapitres finaux concernant respectivement le travail des femmes (son contenu, si l'avis du rapporteur général avait prévalu, se serait intégré à celui des thèmes antérieurs) et le droit du travail et la performance économique. Cette dernière considération est primordiale : "La meilleure des sécurités est [...] celle qui s'appuie sur la création d'emplois" (p. 285).

Les premières démarches consistent donc à mesurer l'écart entre le modèle classique du salariat, à partir duquel s'était édifié un droit du travail tout aussi classique, aujourd'hui fortement remis en cause. "Travail et pouvoir privé" constatent à la fois les progrès de l'autonomie au travail et l'accentuation de la dépendance résultant de la fragmentation du travail. La question centrale est celle, 
bien connue, de la détermination de la frontière du salariat, celle, essentiellement, de la recherche d'un cadre juridique adapté au travail indépendant, qui doit comprendre la situation de l'" entreprise dépendante ". En somme, la perspective est " [...] celle d'un droit commun du travail, dont certaines branches pourraient en revanche ètre adaptées à la diversité des situations de travail [...]" (p. 52). La diversité des situations établies en fonction du marché du travail - formation initiale, contrats d'insertion, chômage, adaptation professionnelle, etc. -, la mobilité du salarié au sein de l'entreprise et entre entreprises incitent à " prendre une vue de l'état professionnel des personnes qui dépasse l'engagement contractuel de leur travail pour embrasser la diversité des formes de travail expérimentées dans la vie humaine " (p. 86) : la notion de travail, "qui déborde l'emploi sans englober la vie tout entière " (p. 88), doit servir d'assise à cet état professionnel. La technique des "droits de tirage sociaux", dont pourrait, par exemple, se réclamer la personne désireuse de se prévaloir d'une période de formation, est avancée. La définition temporelle du travail est en cause. D'une part, observe-t-on, de plus en plus "le temps libre vient se loger dans le temps de travail salarié (congés à des fins de perfectionnement personnel, familiales ou sociales); de l'autre, le travail salarié projette son ombre sur le temps libre (p. 120 et 122)" (pratique des astreintes, du temps partiel annualisé, activités de formation professionnelle, etc.). Dans une perspective d'égalité entre hommes et femmes, "le temps soustrait au travail salarié pour être consacré à des tâches éducatives doit ainsi être conçu comme un moment normal de la carrière des travailleurs, et non comme une rupture dans sa vie professionnelle» (p. 132). La directive européenne du 3 juin 1996 donnant effet à l'accord européen sur le congé parental va d'ailleurs en ce sens.

Par la suite, le rapport se penche plus spécifiquement sur les institutions de régulation du travail. À l'examen des différentes législations nationales en cause, le recours à la négociation collective se généralise ; elle assume ainsi d'une manière plus prononcée des fonctions de flexibilisation des conditions de travail et s'approche davantage de la gestion de l'entreprise. Ce mouvement de décentralisation de la branche vers l'entreprise n'est pas sans susciter de nouveaux problèmes d'articulation des conventions. De nouvelles unités de négociation émergent ; ainsi entrevoit-on d'une façon avantgardiste des modes de négociation engageant des réseaux d'entreprises, notamment sous-traitantes, reliées de façon durable à un même centre donneur d'ouvrage (p. 156). La négociation au niveau communautaire est aussi évoquée. Les systèmes de représentation se transforment eux-mêmes dans une certaine mesure. L'exposé de la situation syndicale dans les divers pays en cause conduit à l'examen de l'évolution possible des systèmes de représentation collective : diversification nécessaire des sujets représentés, compte tenu de celle des situations de travail; des fonctions de représentation aussi qui doivent concilier la défense d'intérêts particuliers et l'intérêt général du travail. Il y va enfin de la réévaluation du rôle de l'État à la lumière des facteurs de changement, qu'il s'agisse notamment de la place de la loi face à la négociation collective, de l'orientation de la sécurité sociale, de l'État-employeur lui-même, de plus en plus en retrait. Émerge globalement un concept de citoyenneté sociale qui "relie les droits sociaux à la notion d'intégration sociale et pas seulement à celle de travail" (p. 306). Contrairement aux analyses néolibérales, dans les sociétés modernes, "c'est l'État qui est le garant du longterme $*$ (p. 230).

Ce rapport, dont le contenu n'a pu ètre ici que bien incomplètement évoqué, tire sa solidité des rapprochements essentiels qu'il opère entre des tendances ou des éléments que l'on oppose souvent facticement : changement et continuité, par 
exemple en ce qui a trait à la représentation collective des salariés; temps de travail et temps familiaux; finalités économiques et finalités sociales, notamment. Il y parvient en proposant des certains concepts catalyseurs du changement et des valeurs véhiculées par le groupe d'experts; ainsi en est-il de l'émergence de la notion d' "état professionnel " des personnes au travail, qui permet de composer avec la flexibilité et la continuité de la vie professionnelle.

Européen par ses préoccupations, $A u$ delà de l'emploi fait état de facteurs de transformation du travail qui paraissent tendre vers du plus universel. Les institutions en cause sont le reflet de différentes traditions nationales, parfois assez différentes de celles qui ont cours en Amérique du Nord. Les valeurs durables qui animent le rapport et ses orientations paraissent, quant à elles, susceptibles de partage entre les deux continents.

PIERRE VERGE Faculté de droit Université Laval

\section{Gestion des ressources humaines: typologies et comparaisons interna- tionales}

par Diane-Gabrielle TREMBlay et David ROLLAND, Sainte-Foy: Télé-université, 1998, 415 p., ISBN 2-7624-1140-8.

En présentant leur ouvrage, les auteurs en formulent d'emblée la visée : comparer divers modèles de gestion des ressources humaines et plus particulièrement les modèles américain, japonais, allemand, suédois et canadien. À l'heure de la mondialisation de l'économie et de la reconnaissance de la diversité culturelle et ethnique dans les entreprises, au moment où de nombreuses firmes canadiennes doivent gérer un personnel croissant à l'étranger, la parution d'un tel ouvrage suscite un intérêt certain.

Mais les objectifs poursuivis par les auteurs débordent les strictes questions de comparaisons. L'ouvrage est utilisé dans un cours de la T'élé-université intitulé "Modèles de gestion des ressources humaines " (ADM 4015). Il importe donc de bien définir les concepts, d'exposer un cadre de référence, de situer la gestion des ressources humaines dans l'environnement plus global des relations industrielles et d'offrir aux étudiants une armature intellectuelle qui leur permette de tirer profit des comparaisons qui suivront.

Cette double nécessité rend l'exercice périlleux et, à prime abord, il n'est pas facile de situer l'ouvrage. Ce n'est pas un manuel classique de GRH et les auteurs s'en défendent. Le praticien ne trouvera pas son compte dans le chapitre 3 consacré aux fonctions de la GRH et le théoricien probablement pas davantage dans le chapitre 2 portant sur les modèles théoriques de la GRH.

Pour bien apprécier l'ouvrage, il faut sans cesse revenir à l'intention des auteurs qui est d'offrir un portrait stylisé des divers modèles de GRH exposés, ainsi que des outils de base permettant de comprendre cette diversité, d'inscrire chaque modèle dans un cadre national, d'évaluer ses forces et ses faiblesses, et de détecter les possibilités d'emprunt d'un pays à l'autre. Et là c'est réussi : pour aborder les divers modèles, le lecteur dispose en effet d'un fil conducteur, se sent constamment guidé, accompagné. La force de cet ouvrage réside dans sa qualité pédagogique, laquelle provient notamment de la cohérence de la démarche suivie pour la présentation de chaque modèle.

Le livre se divise en trois parties : la première partie comprend quatre chapitres qui en constituent l'armature intellectuelle et préparent le lecteur à naviguer dans les eaux internationales. En substance, le lecteur averti y trouvera peu d'éléments nouveaux, si ce n'est au chapitre 4 une 